\title{
Calculation of the Class Numbers of Imaginary Cyclic Quartic Fields
}

\author{
By Kenneth Hardy, ${ }^{*}$ R. H. Hudson, ${ }^{* *}$ D. Richman, \\ Kenneth S. Williams, ${ }^{* * *}$ and N. M. Holtz
}

\begin{abstract}
Any imaginary cyclic quartic field can be expressed uniquely in the form $K=$ $Q(\sqrt{A(D+B \sqrt{D})})$, where $A$ is squarefree, odd and negative, $D=B^{2}+C^{2}$ is squarefree, $B>0, C>0$, and $(A, D)=1$. Explicit formulae for the discriminant and conductor of $K$ are given in terms of $A, B, C, D$. The calculation of tables of the class numbers $h(K)$ of such fields $K$ is described.
\end{abstract}

Let $Q$ denote the field of rational numbers and let $K$ be a cyclic extension of $Q$ of degree 4 . The unique quadratic subfield of $K$ is denoted by $k$. The class number of $K$ (resp. $k$ ) is denoted by $h(K)$ (resp. $h(k)$ ). The conductor of $K$ is denoted by $f=f(K)$. In the case of real cyclic quartic fields $K$, Gras [3] has given a table of the values of $h(K)$ for all such fields with $f<4000$. Recently, the authors have carried out the calculation of the class numbers of imaginary cyclic quartic fields [4]. In this note we give a brief description of the computation of the tables given in [4].

The following explicit representation of a cyclic quartic field is proved in [4, Theorem 1].

THEOREM 1. If $K$ is a real or imaginary cyclic quartic extension of $Q$, then there are integers $A, B, C, D$ such that

$$
K=Q(\sqrt{A(D+B \sqrt{D})})=Q(\sqrt{A(D-B \sqrt{D})}),
$$

where

$$
\left\{\begin{array}{l}
A \text { is squarefree and odd, } \\
D=B^{2}+C^{2} \text { is squarefree, } B>0, C>0 \\
A \text { and } D \text { are relatively prime. }
\end{array}\right.
$$

Moreover, any field satisfying (1) and (2) is a cyclic quartic extension of $Q$. The representation of $K$ in (1) is unique in the sense that if $K=Q\left(\sqrt{A_{1}\left(D_{1}+B_{1} \sqrt{D_{1}}\right)}\right)$ is another representation of $K$, where $A_{1}, B_{1}, C_{1}, D_{1}$ are integers satisfying the conditions of (2), then $D=D_{1}, A=A_{1}, B=B_{1}, C=C_{1}$.

\footnotetext{
Received November 10, 1986.

1980 Mathematics Subject Classification (1985 Revision). Primary 11R29, 11R16; Secondary 11R20.

Key words and phrases. Imaginary cyclic quartic fields, class number, discriminant.

* Research supported by Natural Sciences and Engineering Research Council of Canada Grant A-7823.

** Research supported in part by a research grant from the University of South Carolina.

*** Research supported by Natural Sciences and Engineering Research Council of Canada Grant
} A-7233. 
We remark that the field $K$ is totally real if $A>0$, and is totally imaginary if $A<0$.

Next we let $G$ denote the multiplicative group of residues coprime with $f=f(K)$, so that $G \simeq \operatorname{Gal}\left(Q\left(e^{2 \pi i / f}\right) / Q\right)$. The subgroup of $G$ which is isomorphic to $\operatorname{Gal}\left(Q\left(e^{2 \pi i / f}\right) / K\right)$ is denoted by $H$. $H$ is a subgroup of index 4 in $G$ and the factor group $G / H$ is cyclic of order 4. Let $\alpha$ be an element of $G$ such that $G / H$ is generated by $\alpha H$. We define a character $\chi$ on $G$ by

$$
\chi(\alpha)=i, \quad \chi(h)=1 \text { for all } h \in H .
$$

The character $\chi$ is a quartic, primitive, odd character of conductor $f$.

When $K$ is taken to be an imaginary cyclic quartic field, the class number formula for Abelian fields yields the following formula for $h(K)$ [4, Theorem 3].

THEOREM 2. If $K$ is an imaginary cyclic quartic field of conductor $f$ with quadratic subfield $k$, then

$$
h(K)=\rho h(k)\left|\sum_{0<n<f / 2} \chi(n)\right|^{2},
$$

where the value of the quantity $\rho$ is given by

$$
\rho= \begin{cases}1 / 2, & \text { if } f=5 \\ 1 / 8, & \text { if } f>5, \text { f even } \\ 1 / 2, & \text { if } f>5, \text { fodd }, \chi(2)=1 \\ 1 / 18, & \text { if } f>5, \text { fodd }, \chi(2)=-1 \\ 1 / 10, & \text { if } f>5, \text { fodd }, \chi(2)= \pm i\end{cases}
$$

and $\chi$ is defined in (3).

In order to use Theorem 2 to calculate $h(K)$, we need to be able to compute $f$. A formula for $f$ in terms of $D, A, B, C$ is given in Theorem 3 (see [4, Theorem 5]).

THEOREM 3. The conductor $f(K)$ of the (real or imaginary) cyclic quartic field $K=Q(\sqrt{A(D+B \sqrt{D})})$, where $A$ is squarefree and odd, $D=B^{2}+C^{2}$ is squarefree, $B>0, C>0$, and $(A, D)=1$, is given by

$$
f(K)=2^{\prime}|A| D
$$

where

$$
l= \begin{cases}3, & \text { if } D \equiv 2(\bmod 8) \\ & \text { or } \\ & D \equiv 1(\bmod 4), B \equiv 1(\bmod 2) \\ 2, & \text { if } D \equiv 1(\bmod 4), B \equiv 0(\bmod 2), A+B \equiv 3(\bmod 4) \\ 0, & \text { if } D \equiv 1(\bmod 4), B \equiv 0(\bmod 2), A+B \equiv 1(\bmod 4)\end{cases}
$$

Also, in order to apply (4), it is necessary to be able to compute $\chi(g)$ for $g \in G$. Since $g=\alpha^{i} h$ for some $i=0,1,2,3$ and some $h \in H$, it suffices to have a criterion for recognizing when an element of $G$ is actually in $H$. As each element of $G$ is congruent to a prime modulo $f$, we can test whether $g$ belongs to $H$ or not by means of the following theorem [4, Theorem 6]. 
THEOREM 4. Let $K$ be a (real or imaginary) cyclic quartic field $Q(\sqrt{A(D+B \sqrt{D}})$ ), where $A$ is squarefree and odd, $D=B^{2}+C^{2}$ is squarefree, $B>0, C>0$, and $(A, D)=1$. Let $H$ be the subgroup of the multiplicative group of residues coprime with $f=f(K)$ which is isomorphic to $\operatorname{Gal}\left(Q\left(e^{2 \pi i / f}\right) / K\right)$. Let $p$ be an odd prime not dividing $A, B, C$ or $D$. Then $p \in H$ if and only if

$$
\left(\frac{D}{p}\right)=+1, \quad\left(\frac{A(D+B E)}{p}\right)=+1,
$$

where $D \equiv E^{2}(\bmod p)$ and $(\bar{p})$ denotes Legendre's symbol of quadratic residuacity $(\bmod p)$.

From now on, we restrict $K$ to be an imaginary cyclic quartic field, so that in the representation (1) we have $A<0$. Theorems $1,2,3,4$ were used to calculate the class numbers $h(K)$ of the 3521 distinct fields $K$ with

$$
1<D<1000, \quad 1 \leqslant-A<20,
$$

as well as those of the 4274 fields with conductor $f$ satisfying

$$
f<10,000 \text {. }
$$

The calculations were carried out using computer programs written in PASCAL and implemented on both an IBM micro computer and an APOLLO mini computer. The resulting values are listed in the tables in [4].

We just list at the end of this note the values of $h(K)$ for those fields $K$ with $f \leqslant 200$.

We now describe briefly how the computation of $h(K)$ was carried out. First, Theorem 3 was used to generate two data files containing the values of $(D, A, B, C)$ and $f$ : one for the fields $K$ specified by (9), the other for those fields given by (10). For each of these two data files, a file of the class numbers $h(K)$ was produced as follows.

For each $(D, A, B, C)$ an element $\alpha$ of $G$ was determined such that $G / H=\langle\alpha H\rangle$. This was done by finding a small integer $k$ coprime with $f$ for which the least prime $p \equiv k^{2}(\bmod f)$ satisfied

$$
\begin{aligned}
& \left(\frac{D}{p}\right)=-1, \text { or }\left(\frac{D}{p}\right)=+1\left(\operatorname{say} D \equiv E^{2}(\bmod p)\right) \text { and } \\
& \left(\frac{A(D+B E)}{p}\right)=-1 .
\end{aligned}
$$

By Theorem 4 we see that $p \notin H$. As $|G / H|=4$, we have $(k H)^{4}=H$. Clearly, $(k H)^{2} \neq H$, for otherwise we would have $k^{2} \in H$, contradicting $p \notin H$. Hence, $\operatorname{ord}_{G / H}(k H)=4$ and so we may take $\alpha=k$.

Next, for each ( $D, A, B, C$ ), a set of elements from which the subgroup $H$ is easily constructed, was found. This was done by determining the generators of the cyclic groups of prime power order in the decomposition of $G$ as described, for example, in [1]. These generators were stored together with their orders. The generators of the odd part of $G$ are also the generators of the odd part of $H$. For each generator $g_{i}$ $(i=1,2, \ldots, s)$ of the 2-part of $G$, the unique integer $j\left(g_{i}\right)(=0,1,2,3)$ was determined such that $g_{i} \in \alpha^{j\left(g_{i}\right)} H$, using the value of $\alpha$ calculated above and the criterion of Theorem 4. The values of $j\left(g_{i}\right)$ were stored. The 2-part of $H$ is given by 
the elements

$$
g_{1}^{x_{1}} \cdots g_{s}^{x_{s}} \quad\left(0 \leqslant x_{i} \leqslant \operatorname{ord}\left(g_{i}\right)-1,1 \leqslant i \leqslant s\right)
$$

for which

$$
x_{1} j\left(g_{1}\right)+\cdots+x_{s} j\left(g_{s}\right) \equiv 0(\bmod 4) .
$$

Then, for each ( $D, A, B, C$ ), the value of $\left|\sum_{0<n<f / 2} \chi(n)\right|^{2}$ was calculated by means of the equation

$$
\left|\sum_{0<n<f / 2} \chi(n)\right|^{2}=\left(C_{0}-C_{2}\right)^{2}+\left(C_{1}-C_{3}\right)^{2},
$$

where

$$
C_{r}=\sum_{\substack{0<n<f / 2 \\ n \in \alpha^{\prime} H}} 1 \quad(r=0,1,2,3)
$$

The identities

$$
C_{0}+C_{2}=\phi(f) / 4, \quad C_{1}+C_{3}=\phi(f) / 4
$$

served as checks on the calculation.

As the relative class number $h(K) / h(k)$ is an integer, the following congruence holds (see Theorem 2):

$$
\left(C_{0}-C_{2}\right)^{2}+\left(C_{1}-C_{3}\right)^{2} \equiv 0(\bmod t)
$$

where

$$
t= \begin{cases}8, & \text { if } f>5, f \text { even } \\ 18, & \text { if } f>5, f \text { odd }, \chi(2)=-1 \\ 10, & \text { if } f>5, f \text { odd, } \chi(2)= \pm i \\ 2, & \text { otherwise }\end{cases}
$$

This congruence was used as another check on the calculation, in order to reduce the chances of a computer error.

Finally, Theorem 2 was used to calculate $h(K)$ from the values of $\left(C_{0}-C_{2}\right)^{2}+$ $\left(C_{1}-C_{3}\right)^{2}$, the values of $h(k)$ given in [6], and the values of $\rho$ defined by (5).

When $D=q($ prime $)=a^{2}+b^{2} \equiv 5(\bmod 8), q<1000, A=-1, B=b \equiv 0$ $(\bmod 2), C=a \equiv 1(\bmod 2)$, our values agree with those in [5]. In addition, when $D=5$, our values agree with those which can be deduced from the table in [2].

The following table is a very short extract taken from the second table in [4].

Table of class numbers of imaginary cyclic quartic fields

$$
Q(\sqrt{A(D+B \sqrt{D})}),
$$

where $D, A, B, C$ are integers such that

$A$ is squarefree, odd, and negative,

$$
\begin{aligned}
& D=B^{2}+C^{2} \text { is squarefree, } B>0, C>0, \\
& (A, D)=1
\end{aligned}
$$

in the range

$$
f<200 \text {. }
$$




\begin{tabular}{|c|c|c|c|c|c|c|c|}
\hline case & $f$ & $D$ & $-A$ & $B$ & $C$ & $h(k)$ & $h(K)$ \\
\hline 1 & 5 & 5 & 1 & 2 & 1 & 1 & 1 \\
\hline 2 & 13 & 13 & 1 & 2 & 3 & 1 & 1 \\
\hline 3 & 16 & 2 & 1 & 1 & 1 & 1 & 1 \\
\hline 4 & 29 & 29 & 1 & 2 & 5 & 1 & 1 \\
\hline 5 & 37 & 37 & 1 & 6 & 1 & 1 & 1 \\
\hline 6 & 40 & 5 & 1 & 1 & 2 & 1 & 2 \\
\hline 7 & 48 & 2 & 3 & 1 & 1 & 1 & 2 \\
\hline 8 & 51 & 17 & 3 & 4 & 1 & 1 & 10 \\
\hline 9 & 53 & 53 & 1 & 2 & 7 & 1 & 1 \\
\hline 10 & 60 & 5 & 3 & 2 & 1 & 1 & 4 \\
\hline 11 & 61 & 61 & 1 & 6 & 5 & 1 & 1 \\
\hline 12 & 65 & 5 & 13 & 2 & 1 & 1 & 2 \\
\hline 13 & 65 & 13 & 5 & 2 & 3 & 1 & 2 \\
\hline 14 & 68 & 17 & 1 & 4 & 1 & 1 & 4 \\
\hline 15 & 80 & 2 & 5 & 1 & 1 & 1 & 2 \\
\hline 16 & 80 & 10 & 1 & 1 & 3 & 2 & 20 \\
\hline 17 & 80 & 10 & 1 & 3 & 1 & 2 & 4 \\
\hline 18 & 85 & 5 & 17 & 2 & 1 & 1 & 2 \\
\hline 19 & 85 & 85 & 1 & 2 & 9 & 2 & 20 \\
\hline 20 & 85 & 85 & 1 & 6 & 7 & 2 & 4 \\
\hline 21 & 101 & 101 & 1 & 10 & 1 & 1 & 5 \\
\hline 22 & 104 & 13 & 1 & 3 & 2 & 1 & 2 \\
\hline 23 & 105 & 5 & 21 & 2 & 1 & 1 & 4 \\
\hline 24 & 109 & 109 & 1 & 10 & 3 & 1 & 17 \\
\hline 25 & 112 & 2 & 7 & 1 & 1 & 1 & 4 \\
\hline 26 & 119 & 17 & 7 & 4 & 1 & 1 & 2 \\
\hline 27 & 120 & 5 & 3 & 1 & 2 & 1 & 4 \\
\hline 28 & 123 & 41 & 3 & 4 & 5 & 1 & 34 \\
\hline 29 & 136 & 17 & 1 & 1 & 4 & 1 & 4 \\
\hline 30 & 140 & 5 & 7 & 2 & 1 & 1 & 4 \\
\hline 31 & 145 & 5 & 29 & 2 & 1 & 1 & 4 \\
\hline 32 & 145 & 29 & 5 & 2 & 5 & 1 & 4 \\
\hline 33 & 149 & 149 & 1 & 10 & 7 & 1 & 9 \\
\hline 34 & 156 & 13 & 3 & 2 & 3 & 1 & 8 \\
\hline 35 & 157 & 157 & 1 & 6 & 11 & 1 & 5 \\
\hline 36 & 164 & 41 & 1 & 4 & 5 & 1 & 4 \\
\hline 37 & 165 & 5 & 33 & 2 & 1 & 1 & 8 \\
\hline 38 & 173 & 173 & 1 & 2 & 13 & 1 & 5 \\
\hline 39 & 176 & 2 & 11 & 1 & 1 & 1 & 10 \\
\hline 40 & 181 & 181 & 1 & 10 & 9 & 1 & 25 \\
\hline 41 & 185 & 5 & 37 & 2 & 1 & 1 & 10 \\
\hline 42 & 185 & 37 & 5 & 6 & 1 & 1 & 10 \\
\hline 43 & 187 & 17 & 11 & 4 & 1 & 1 & 34 \\
\hline 44 & 195 & 65 & 3 & 8 & 1 & 2 & 8 \\
\hline 45 & 195 & 65 & 3 & 4 & 7 & 2 & 104 \\
\hline 46 & 197 & 197 & 1 & 14 & 1 & 1 & 5 \\
\hline
\end{tabular}

K. Hardy and K. S. Williams

Department of Mathematics and Statistics Carleton University

Ottawa, Ontario, Canada K1S 5B6

R. H. Hudson and D. Richman

Department of Mathematics

University of South Carolina

Columbia, South Carolina 29208

N. M. Holtz

Department of Civil Engineering

Carleton University

Ottawa, Ontario, Canada K1S 5B6 
1. E. D. Bolker, Elementary Number Theory, Benjamin, New York, 1970.

2. HaRvey COHN, "A computation of some bi-quadratic class numbers," MTAC, v. 12, 1958, pp. 213-217.

3. M.-N. Gras, Table Numérique du Nombre de Classes et des Unités des Extensions Cycliques Réelles de Degré 4 de Q, Publ. Math. Univ. Besançon, 1977/78, fasc. 2, 53 pp.

4. K. Hardy, R. H. Hudson, D. Richman, K. S. Williams \& N. M. Holtz, Calculation of the Class Numbers of Imaginary Cyclic Quartic Fields, Carleton-Ottawa Mathematical Lecture Note Series, No. 7, July 1986, $201 \mathrm{pp}$.

5. R. H. Hudson \& K. S. Williams, A Class Number Formula for Certain Quartic Fields, Carleton Mathematical Series No. 174, February 1981, 25 pp.

6. B. ORIAT, Groupe des Classes des Corps Quadratiques Réels $Q(\sqrt{d}), d<10000$, Faculté des Sciences de Besançon, Besançon, France, 53 pp. 